\title{
Article
}

\section{Integration of mental health screening and treatment into cystic fibrosis clinics: Evaluation of initial implementation in 84 programs across the United States}

Quittner, A, Abbott, Janice, Hussain, S, Ong, T, Uluer, A, Hempstead, S, Lomas, P and Smith, B

Available at http://clok.uclan.ac.uk/34145/

Quittner, A, Abbott, Janice ORCID: 0000-0001-9851-1236, Hussain, S, Ong, T, Uluer, A, Hempstead, S, Lomas, P and Smith, B (2020) Integration of mental health screening and treatment into cystic fibrosis clinics: Evaluation of initial implementation in 84 programs across the United States. Pediatric Pulmonology . ISSN 8755-6863

It is advisable to refer to the publisher's version if you intend to cite from the work. http://dx.doi.org/10.1002/ppul.24949

For more information about UCLan's research in this area go to http://www.uclan.ac.uk/researchgroups/ and search for <name of research Group>.

For information about Research generally at UCLan please go to http://www.uclan.ac.uk/research/

All outputs in CLoK are protected by Intellectual Property Rights law, including Copyright law. Copyright, IPR and Moral Rights for the works on this site are retained by the individual authors and/or other copyright owners. Terms and conditions for use of this material are defined in the policies page. 
Integration of Mental Health Screening and Treatment into Cystic Fibrosis Clinics :

Evaluation of Initial Implementation in 84 Programs Across the United States

Alexandra L. Quittner, PhD; Janice Abbott, PhD; Saida Hussain, PhD; Thida Ong, MD; Ahmet Uluer, DO MPH; Sarah Hempstead, MS; Paula Lomas, MAS, RN; \& Beth Smith, MD

Keywords: dissemination, implementation science, barriers, depression screening, anxiety screening, caregiver screening 


\begin{abstract}
Background: A large-scale epidemiological study of 6088 individuals with CF and 4102 caregivers in 9 countries documented elevated symptoms of depression and anxiety, leading to international guidelines for annual screening and follow-up. To facilitate national implementation, $84 \mathrm{CF}$ programs funded a mental health coordinators (MHC). Implementation was evaluated after 1 year using the Consolidated Framework for Implementation Research (CFIR) to identify facilitators and barriers.
\end{abstract}

Methods: A 45-item internet survey was developed to assess relevant CFIR implementation steps. Surveys were completed in 2016. It assessed five domains tailored to study aims: 1) Intervention Characteristics, 2) Outer Setting, 3) Inner Setting, 4) Characteristics of Individuals, and 5) Process of Implementation.

Results: Response rate was $88 \%$, with pediatric and adult programs equally represented. A majority of MHCs were social workers (54.1\%) and psychologists (41.9\%); 41\% had joined the team in the past year. Facilitators across the 5 domains included universal uptake of screening tools, greater awareness and detection of psychological symptoms, reduced stigma, and positive feedback from patients and families. Barriers included limited staff time, space, and logistics.

Discussion: This is the largest systematic effort to integrate mental health screening and treatment into the care of individuals with a serious, chronic illness and their caregivers. MHCs implementing screening, interpretation and follow-up reported positive results, and significant barriers. This national implementation effort demonstrated that depression and anxiety can be efficiently evaluated and treated in a complex, chronic disease. Future efforts include recommending the addition of screening scores to national CF Registries and examining their effects on health outcomes. 


\section{Introduction}

A large-scale epidemiological study of the prevalence of depression and anxiety in individuals with cystic fibrosis (CF) and parent caregivers provided clear evidence of elevated risk ${ }^{1}$. Across nine countries, $17 \%$ of the 6088 patients, ages 12 through adulthood, reported elevated symptoms of depression and 30\% reported elevated symptoms of anxiety (TIDES study; The International Depression/Anxiety Epidemiological Study ${ }^{1}$ ). Rates of depression and anxiety among the 4102 parent caregivers ranged from 31-48\%. Across respondents, rates of depression and anxiety were two to three times higher than community samples ${ }^{2-4}$. High comorbidity of these psychological symptoms was also documented across 1122 parent-teen dyads, with adolescents more than twice as likely to report elevated symptoms of depression or anxiety if either parent was elevated, indicating that a family systems approach to addressing mental health is needed.

Depression and anxiety are a major concern because they are strongly associated with negative health consequences and outcomes: worse adherence ${ }^{5-12}$, worse pulmonary function and body mass index ${ }^{8,13,14}$, decreased health-related quality of life 6,7 , and increased hospitalizations and healthcare $\operatorname{costs}^{14}$. Depression was associated with a higher risk of mortality within 5 years ${ }^{15}$. Further, new studies in other chronic conditions suggest that depression, independent of disease, is associated with inflammation $^{16}$. Given that inflammation in CF has been linked to recurring pulmonary exacerbations, which are associated with greater morbidity and earlier mortality ${ }^{16}$, addressing mental health issues may be an important pathway to improving health and extending lifespan in $\mathrm{CF}$.

The TIDES study was the major impetus for integrating mental health into the specialized medical care for people with $\mathrm{CF}$, given that rates of depression and anxiety were 2-3 times higher than those found in community samples ${ }^{1}$. Thus, these results led the Cystic Fibrosis Foundation (CFF) and the European Cystic Fibrosis Society (ECFS) to form an International Committee on Mental Health in CF (ICMH). A representative, multidisciplinary group of $\mathrm{CF}$ experts (including a person with $\mathrm{CF}$, two caregivers) met for 
two years to develop guidelines for the screening and treatment of depression and anxiety in both individuals with CF and parent caregivers ${ }^{17}$. The ICMH's recommendations included standardized screening instruments, follow-up, and evidence-based recommendations for psychological and pharmacological treatments. Although depression screening has been recommended by other national associations (e.g., American Diabetes Association ${ }^{18}$, American Academy of Pediatrics ${ }^{19}$ ), few of these guidelines have been implemented ${ }^{20}$, and none have recommended screening both adolescents with a chronic illness and their caregivers. A systematic review examining evidence on improving the quality of mental health care delivery identified a dearth of studies that were successful with this integration and organizational change ${ }^{21}$.

To facilitate implementation of these guidelines, a competitive grant process was initiated by the CFF to fund a Mental Health Coordinator (MHC) for 3 years, with a primary role of initiating screening according to the guidelines, coordinating follow-up, and tracking outcomes across 84 CF programs. Grants were peer-reviewed by a 15-member multidisciplinary committee. All CFF-accredited Care Center, Programs and Affiliates were eligible to apply $(n=277)$. CFF received 154 grant proposals which were judged on the following criteria: 1) Current progress in implementing mental health screening in their clinic; 2) Plans to implement screening and treatment; 3) Plans to make referrals for mental health services in their hospital and community; 4) Plans to provide psychological support and interventions as needed; and 5) Plan for sustainability. We were not able to include CF Centers in other countries. However, a number of international implementation efforts were undertaken once the guidelines were implemented in Year 1. A member of the CFF Mental Health Advisory Committee serves as a liaison to the European Cystic Fibrosis Society to facilitate dissemination of the guidelines in Europe, another member of this committee trained $10 \mathrm{CF}$ Centers in Canada, and two committee members launched a “Mental Health Roadshow” across Australia. 
To evaluate the success of this implementation effort, the Consolidated Framework for Implementation Research ${ }^{22}$ (CFIR) was utilized and customized to reflect the new psychological screening and treatment guidelines. CFIR is the most widely used conceptual framework, with over 300 published articles, which assesses stakeholder's perceptions of the benefits of a new intervention strategies (i.e., mental health screening of people with CF and caregivers), as well as the key facilitators and barriers of intervention success. Thus, it was designed to lead to iterative improvements in delivery of a new healthcare intervention over time.

Although the CFIR model is considered the "gold standard" framework for evaluating implementation of new practice guidelines, it is complex and cumbersome, and requires tailoring of the key variables to the specific context ${ }^{23}$. Five primary domains were modeled: 1) Intervention Characteristics, 2) Outer Setting, 3) Inner Setting, 4) Characteristics of Individuals, and 5) Process of Implementation (see Figure 1).

To evaluate implementation success, we tailored the five CFIR domains to the aims of the published mental health guidelines and defined each implementation construct and its relevant facilitators and barriers (see Table 1). The first domain, Intervention Characteristics, is characterized by implementation of annual mental health screening, provision of brief interventions in clinic, and referrals into the community. The second domain, Outer Setting, includes evidence-based guidelines, CFF resources and national conference, widespread adoption of the guidelines. The third domain, Inner Setting, is exemplified by CF team culture, training in quality improvement, CF Center Director and CFF Executive Leadership. The fourth domain, Characteristics of individuals, includes professional training of MHC, years of experience in CF care, and individual training opportunities at the North American Cystic Fibrosis Conference (NACFC). The final domain, Process of Implementation, is embodied by identification of the MHC champion, involvement of stakeholders on the team, and plan for execution of annual screening and follow-up. Thus, the major aim of this study was to apply the CFIR constructs 
defined above to evaluate the implementation of standardized screening of depression and anxiety among individuals with CF and primary caregivers, and secondarily, gathered information to improve this process.

\section{Methods}

\section{Participants and procedure}

CFF administered the internet survey (via SurveyMonkey) to the first $84 \mathrm{CF}$ pediatric and adult programs that received the award, with a start-date of January, 2016. The survey was emailed to the principal investigator (i.e., Center Director) and distributed on September $7^{\text {th }} 2016$, with reminders sent November 3, 10 and December 2. Thus, many programs had less than one year of implementation experience.

\section{Measure}

The survey was developed by the CFF-sponsored Mental Health Advisory Committee (MHAC), a multidisciplinary research subcommittee comprised of health care professionals (e.g., pulmonologists, psychologists, psychiatrists, adult with CF) with strong interests in advancing integration of mental health into CF care. Seven US and one UK member developed the survey. An iterative, consensus-based process was used to create the 45-items (see online supplement), organized into the five CFIR domains described above. Most responses were 'check boxes' or required numerical data (e.g. characteristics of center, screening outcomes), with 1 open-ended question on major successes.

\section{Data analysis}

Data were analyzed using SPSS version 24. Multiple response frequencies and the mean rank order of items were determined. Text responses were analyzed thematically and a rigorous method of coding the text was devised ${ }^{24}$. Line by line coding of the transcripts was undertaken, with codes attached to specific words, phrases, or sentences. These codes were then grouped into conceptual categories, based on emerging themes (coherent, meaningful patterns in the data). This was done independently by two 
researchers $(\mathrm{JA}, \mathrm{LV})$ to ensure that comparable interpretations were being made. When discrepancies were found, they were discussed and resolved through consensus.

\section{Results}

There were 74 respondents, yielding a response rate of $88 \%$. Not all programs had hired or integrated an MHC into their program when the survey was deployed. Table 2 reflects aspects of the Inner Setting and Characteristics of the Individuals. A majority of programs employed a social worker (40 of 74 programs, $54.1 \%$ ) or psychologist (41.9\%) to perform mental health screening and follow-up. Approximately half of these professionals were newly employed or had been integrated into the CF team for less than 1 year (38 of 74 programs, 51\%). We were not able to document when and how these positions were filled; grants were awarded at the same time, but the contracting process often varied across hospitals and medical centers.

\section{1) Screening process and use of the recommended screening tools}

Results of the screening process are presented in Table 3 and focused on questions related to the processes of implementation. This table, which reports the percentage of MHCs who endorsed items on: 1) preparedness for screening patients and caregivers; 2) ease of administering, scoring and interpreting the screening scores; 3) MHC observations of patient and caregiver perceptions of screening and 4) MHC training and ability to provide follow-up and referrals. These results reflect both Intervention Characteristics and Process of Implementation.

\section{2) Successes and barriers to implementation}

The emerging themes concerning 'successes' are highlighted in Table 4. They demonstrate: 1) greater awareness, detection and management of MH issues by CF professionals, "prevention and early identification before major mental health concerns arise," 2) reductions in stigma and willingness to discuss MH issues, "increased normalization of mental health concerns in CF," and 3) gratitude and support from families / patients for addressing $\mathrm{MH}$, "most families are very excited about this and find 
mental health a much needed addition in the clinic." MHCs reported that numerous patients and caregivers expressed their appreciation that $\mathrm{MH}$ is being measured and addressed, and they noted their increased understanding of 'treatment' options available for $\mathrm{MH}$ problems. These results reflect Intervention Characteristics, Outer Setting, and Inner Setting.

Respondents were asked to rank a list of nine potential barriers to screening (from most to least significant). The 'top' barriers across respondents were: 1) limited staff time (53\%), space limitations (47\%), perceived patient burden (42\%) and logistics e.g., storing data (42\%). A sub-analysis compared the perceived barriers reported by social workers vs. psychologists/psychiatrists and found that social workers ranked "patient burden" as the top barrier, whereas psychologists/psychiatrists ranked "limited staffing" as the top barrier, with "patient burden" ranked $4^{\text {th }}$. Additionally, data generated from the thematic analysis highlighted the main barriers/ethical dilemmas faced by MHCs (Table 5): 1) the 'ethical and legal' aspects of screening adult caregivers in a pediatric department and storage of caregiver data and 2) difficulty with logistics, follow-up and referrals. Additional resources rated as most helpful were: 1) more mental health staff, in particular psychiatrists, and 2) support outside the clinic, e.g. community providers. These results reflect both Intervention Characteristics and Inner Setting.

\section{3) Patient and caregiver perceptions and outcomes}

Programs reported variable start times for initiating screening. Some programs had already begun setting up these processes and others had started only a few months prior to the survey. Approximately 5000 individuals and over 1000 caregivers were screened. Numerical results of individual patients per program were not obtained given the quality improvement focus of this survey. Suicidal ideation was endorsed by 141 patients and 15 caregivers. Figure 2 provides the data on referrals and interventions. This reflects the Process of Implementation and Intervention Characteristics.

\section{4) Needs assessment focused on additional education and training for MHCs.}


Only 2 programs reported they did not have a team member trained in MH counseling interventions. Respondents reported participating in: training in $\mathrm{MH}$ screening (91.9\%), cognitive behavioral therapy (85.1\%), counselling or supportive therapy (94.6\%) and prescribing medications for depression/anxiety (44.6\%). A sub-analysis compared providers' perceptions of how adequately trained they were in counselling those scoring in the mild range: $60 \%$ of social workers rated their training as "adequate," whereas $91 \%$ of psychologists/psychiatrists rated it as "adequate," $\left.\mathrm{X}^{2}(3, \mathrm{~N}=77)=12.42, \mathrm{p}<.01\right)$. For those scoring in the moderate to severe range, only $40 \%$ of social workers felt adequately trained to provide counselling compared to $88 \%$ of psychologists/psychiatrists $\left(\mathrm{X}^{2}(3, \mathrm{~N}=77)=18.56, \mathrm{p}<.001\right)$. Chi Square analyses were also utilized to compare responses to the question "How easy has it been to implement screening?" for the variables related to length of experience working on a CF Team and pediatric vs. adult CF programs. There were no differences in ease of implementation based on years of experience on a CF Team $\left(X^{2}(9, n=74)=7.91, p=.59, n s\right)$, We also found no differences in responses to this question based on whether the CF program served pediatric or adult patients with CF (e.g., we removed CF Centers that care for both populations) $\left(X^{2}(3, n=51)=3.70, p=.29, n s\right)$. The following education and training programs were endorsed by respondents: 1) a workshop on the screening process $(67.6 \%), 2)$ access to a $\mathrm{MH}$ hotline $(60.8 \%), 3)$ short course on medications $(44.9 \%), 4)$ access to a mentoring program (44.5\%) and 5) short courses on Cognitive Behavioral Therapy (CBT) and Interpersonal therapy (36.5\%). These results reflect Intervention Characteristics, Process of Implementation, and the Outer Setting.

\section{Discussion}

This is the largest systematic effort ever conducted to integrate mental health screening and treatment into the care of individuals with a serious, chronic illness and their caregivers. Over 5,000 individuals with CF and over 1,000 caregivers were screened in one year. Our adaptation of the CFIR model was comprised of: 1) an international epidemiological study of the prevalence of depression and 
anxiety $\left.^{1}, 2\right)$ a review of the literature by experts in the US and Europe to identify the best screening tools and develop evidence-based guidelines for screening and psychological/ pharmacological treatments, 3) implementation of a standardized screening and follow-up protocol, and 4) implementation grants provided to $84 \mathrm{CF}$ programs in the US. At the end of Year 1, MHCs completed a survey evaluating both the implementation process, its successes, barriers, and their future educational and training needs. Both rating scale and qualitative responses were analyzed.

Dissemination and implementation of guidelines have always faced significant challenges, in part because changes to health care systems and practices are so complex ${ }^{25}$. However, with the support of the CFF, efforts by the MHAC and strategic planning, we demonstrated positive results in implementing these guidelines. The response rate was $88 \%$, which was excellent, given that programs were in the hiring or orientation process. The sample was diverse and nationally representative, with programs ranging from small to large, located across the US, and similar to programs accredited by CFF. Overwhelmingly positive results were found in preparation to conduct mental health screening and use and interpretation of the screening tools. Patients and caregivers were perceived as positive about the screening process (over 95\%) and most MHCs rated their training as "somewhat adequate-very adequate." An unanticipated benefit of this implementation effort was the introduction of new mental health professionals (e.g., social workers, psychologists, psychiatrists) to the multidisciplinary CF team. Over half of the MHCs were either new to $\mathrm{CF}$ or had been working in this area for less than one year, indicating that new professionals joined CF programs, resulting in an influx of new talent, energy and ideas. Providers who were new to CF Care did not find implementation more difficult than those with many years of CF experience.

One overarching implementation challenge in the Intervention Characteristics domain was preparing MHCs to address suicidal ideation if endorsed on the PHQ-9. We recommended training in the Columbia Suicide Severity Rating Scale (CSSRS) ${ }^{26}$ and use of this assessment tool if question \#9 is endorsed. The CSSRS has extensive validation data and is used by both healthcare (doctors, nurses) and 
lay professionals (e.g., policeman, teachers), followed by a clinical interview. Training on the CSSRS yielded an online training certificate. Endorsement of this item occurred more frequently than we anticipated (results above), which affirmed our decision to include this item in screening. CF Centers were also encouraged to develop suicide safety plans that could be activated if needed.

A second implementation challenge, documented in the Inner Setting, was initiating screening for caregivers. This recommendation, to screen depression/anxiety in parents of children with CF ages birth to 17 years, was controversial given that parents are not the "identified patient." Although the ICMH had empirical data to support this recommendation (i.e., high prevalence of elevated symptoms across over 4,000 caregivers $^{17}$ ), a number of concerns were raised prior to implementation: 1) ethical issues related to screening parents, 2) recording and storing their data, and 3) challenges referring caregivers for psychological assistance when needed. Our implementation data shed light on these issues. Although caregivers were very positive about screening (over 95\% "somewhat-very positive"); and most MHCs were "somewhat" to "very prepared" to screen parents, and follow-up was "somewhat" to "very easy," challenges remained. These included ethical issues about reporting and storing caregiver data, and having adequate space to screen adolescents separately from their parents ${ }^{20,27}$. In general, MHCs were successful in screening parents, which enabled these pediatric programs to take a "family systems" approach to managing CF. Given the high rates of depression and anxiety reported by parents ${ }^{5,17}$ and the importance of acknowledging and validating this as a normative response, our implementation effort could serve as a model for providing more family-centered care in other pediatric specialty clinics (e.g., diabetes, sickle cell, epilepsy).

The most commonly reported barriers were: limited staff time, space limitations, respondent burden, and logistics (e.g., storing data), endorsed by $42 \%-53.4 \%$ of MHCs. Many of these barriers seemed solvable, and establishment of the mental health listserv led to frequent discussions of 
these barriers and potential solutions ${ }^{1}$. The MHAC has encouraged processes that increase efficiency, such as use of electronic screening (e.g., iPads, tablets) with automatic scoring and storage using programs, such as RedCap, which is HIPPA compliant and secure, rather than paper-pencil data collection, which requires data entry and storage. Screening takes 5-8 minutes with the PHQ-9 and GAD7, but if scores are elevated, additional time is required to discuss next steps. Solving logistics related to space is more difficult. Space is at a premium in most CF clinics, and infection control guidelines for people with $\mathrm{CF}^{28}$ recommend immediate placement in an exam room, with disinfection between patients. Use of disposable Ziploc bags for screening on iPads or phones has also been widely implemented for infection control. The psychosocial listserv is open to everyone, with about 425 participants from 10-12 countries. To address training needs, the CFF and MHAC worked together to provide a toolbox and Welcome Packet for MH screening², workshops and short courses at the annual North American Cystic Fibrosis Conference (NACFC), and a new, phone-based mentoring program. These links and tools are available to everyone.

Implementation of new procedures and quality improvement in any learning health care system requires an iterative process to solve emerging barriers and identify the key successes ${ }^{29}$. The benefits of this screening program were significant, including earlier and better identification of mental health symptoms, reductions in the stigma related to these symptoms, improved understanding of the behavioral health variables that affect disease management and health outcomes (e.g., depression leading to worse adherence), and greater collaboration within the multidisciplinary team. Critically important, improvements in symptoms of depression and anxiety were observed by MHCs and appropriate interventions were provided to the 141 individuals with $\mathrm{CF}$ and 15 caregivers who endorsed suicidal

\footnotetext{
${ }^{1}$ To join the mental health listserv, visit mentalhealth@cff.org

${ }^{3}$ To access training materials, visit mentalhealth@cff.org
} 
ideation. There is little data on the prevalence of suicidal ideation or explicit suicide in the CF population, but it is likely much higher than realized.

In general, MHCs reported they were prepared and well-trained; 92\% attended a training and 85\% had training in CBT interventions. More training was needed in prescribing anti-depressant medications (64\%) and MHCs requested access to a mental health hotline and mentoring (44.5\%). These training needs have been targeted in the NACFC meeting via short courses, symposia and "brown bag" sessions. The success of this mental health implementation effort, including nearly universal uptake of screening, is also testament to the dedication of CF care teams in integrating mental health into routine care, and their recognition of the importance of normalizing and addressing mental health concerns.

Limitations and Future Directions

This large-scale implementation effort at 84 pediatric and adult CF programs was an ambitious project guided by our CFIR model. The CF Foundation provided funding to hire new mental health professionals or covered the time of existing staff to conduct screening and follow-up via this competitive grant mechanism. This financial support clearly jump-started the process, however the task now is to sustain it when current funding ends. The difficulties of sustaining these system changes underlies every new initiative and quality improvement project that seeks to improve the delivery of health care, particularly for complex, chronic conditions ${ }^{29}$. A number of avenues for sustainability are being explored: 1) billing for psychological services, 2) financial savings associated with treating mental health concerns, potentially reducing the costs of poor adherence, missed clinic visits, and hospitalizations, and 3) developing cost-sharing models with hospital administrators. The Cystic Fibrosis Foundation is committed to supporting the integration of mental health into the specialized care provided to people with CF and plans to provide additional funding beginning mid-year. Ultimately, sustaining this program will require advocacy by those who are affected--individuals with $\mathrm{CF}$, caregivers, and providers serving this population - to speak out about the importance of integrating mental health and physical care, and 
lobbying for continuation of these efforts. Future research should evaluate the effectiveness of this mental health intervention on clinic attendance, adherence, frequency of hospitalizations, health-related quality of life, and mortality ${ }^{15}$. One critical avenue for advancing this process is to recommend inclusion of these screening scores in well-established national registries (e.g., UK and US). These data will be critical to the sustainability of this implementation model.

Additional limitations of this study should be noted. The survey required self-reported responses, and there is always the potential for self-report data to be biased, depending on the perspective of the respondent. However, it is critical to evaluate the perceptions of the professionals who are implementing the mental health screening protocol. The alternative, collecting observational data, was not feasible in a study surveying this large number of CF programs. Given that this was the first year these grants were awarded, the initial Year 1 survey may have recorded more positive results, skewed by the novelty of this mental health initiative (e.g., a honeymoon period). On the other hand, given the challenges of initiating any systematic change in a health care system, the MHCs may have also been experiencing "growing pains" as they identified the best processes for conducting screening and brief interventions.

Further, not all CF programs were funded to initiate screening, and thus, these data do not represent all US CF programs. However, 36 additional CF programs were funded in a second request for applications, and a $3^{\text {rd }}$ round funded 18 more CF Centers. Furthermore, the CFF has made an additional commitment to provide ongoing funding to all $\mathrm{CF}$ programs for mental health screening and treatment. Given that this study was not focused on collecting and analyzing screening data from patients and caregivers, we could not demonstrate that identifying and treating these psychological symptoms made a difference in health outcomes. Adding screening data to existing registries would be required for this.

In conclusion, we have shown that it is possible to incorporate mental health screening and treatment into busy, specialized clinics that treat a complex, chronic illness that begins at birth and continues through adulthood. Several implementation efforts contributed to this success, including 
development of a toolkit to help practitioners, a listserv that became an open forum for providers in the US and other countries to post questions and offer suggestions, and the incorporation of training sessions into the annual CF conference, which built expertise into the national community. This mental health screening and treatment program can serve as a model for patient-centered care in other chronic conditions, many of which also have a high prevalence of depression and anxiety (e.g., multiple sclerosis, epilepsy), that is not currently addressed ${ }^{30,31}$. This project has now expanded screening to 138 programs, and data are being gathered now to track this implementation process over time.

\section{Acknowledgements}

The Cystic Fibrosis Foundation has supported the activities of the Mental Health Advisory Committee, including group meetings. We gratefully acknowledge the valuable input of Dr. Emily Muther, Colorado Children's Hospital, and Laura Tillman of Ann Arbor, Michigan. We also thank the participants for taking the time to complete this survey.

\section{References}

1. Quittner AL, Goldbeck L, Abbott J, Duff A, Lambrecht P, Solé A, et al. Prevalence of depression and anxiety in patients with cystic fibrosis and parent caregivers: results of The International Depression Epidemiological Study across nine countries. Thorax. 2014 Dec;69(12):1090-7.

2. Martin A, Rief W, Klaiberg A, Braehler E. Validity of the Brief Patient Health Questionnaire Mood Scale (PHQ-9) in the general population. Gen Hosp Psychiatry. 2006 Feb;28(1):71-7.

3. Pignone MP, Gaynes BN, Rushton JL, Burchell CM, Orleans CT, Mulrow CD, et al. Screening for depression in adults: a summary of the evidence for the U.S. Preventive Services Task Force. Ann Intern Med. 2002 May 21;136(10):765-76.

4. Dierker LC, Albano AM, Clarke GN, Heimberg RG, Kendall PC, Merikangas KR, et al. Screening for anxiety and depression in early adolescence. J Am Acad Child Adolesc Psychiatry. 2001 Aug;40(8):92936.

5. Smith BA, Modi AC, Quittner AL, Wood BL. Depressive symptoms in children with cystic fibrosis and parents and its effects on adherence to airway clearance. Pediatr Pulmonol. 2010 Aug;45(8):756-63.

6. Havermans T, Colpaert K, Dupont LJ. Quality of life in patients with Cystic Fibrosis: association with anxiety and depression. J Cyst Fibros Off J Eur Cyst Fibros Soc. 2008 Nov;7(6):581-4. 
7. Riekert KA, Bartlett SJ, Boyle MP, Krishnan JA, Rand CS. The association between depression, lung function, and health-related quality of life among adults with cystic fibrosis. Chest. 2007 Jul;132(1):2317.

8. Yohannes AM, Willgoss TG, Fatoye FA, Dip MD, Webb K. Relationship between anxiety, depression, and quality of life in adult patients with cystic fibrosis. Respir Care. 2012 Apr;57(4):550-6.

9. Latchford G, Duff AJA. Screening for depression in a single CF centre. J Cyst Fibros. 2013 Dec $1 ; 12(6): 794-6$.

10. Besier T, Born A, Henrich G, Hinz A, Quittner AL, Goldbeck L, et al. Anxiety, depression, and life satisfaction in parents caring for children with cystic fibrosis. Pediatr Pulmonol. 2011 Jul;46(7):672-82.

11. Costello, Jane E, Erkanli A, Angold A. Is there an epidemic of child or adolescent depression? J Child Psychol Psychiatry. 2006 Dec;47(12):1263-71.

12. Grenard JL, Munjas BA, Adams JL, Suttorp M, Maglione M, McGlynn EA, et al. Depression and medication adherence in the treatment of chronic diseases in the United States: a meta-analysis. J Gen Intern Med. 2011 Oct;26(10):1175-82.

13. Goldbeck L, Besier T, Hinz A, Singer S, Quittner AL, TIDES Group. Prevalence of symptoms of anxiety and depression in German patients with cystic fibrosis. Chest. 2010 Oct;138(4):929-36.

14. Snell C, Fernandes S, Bujoreanu IS, Garcia G. Depression, illness severity, and healthcare utilization in cystic fibrosis. Pediatr Pulmonol. 2014 Dec;49(12):1177-81.

15. Schechter M, Ostrenga J, Fink A, Barker D, Sawicki G, Quittner A. Five year surivival is decreased in CF patients screening positive for depression. Pediatr Pulmonol. 2017;52:404-5.

16. Cepeda MS, Stang P, Makadia R. Depression Is Associated With High Levels of C-Reactive Protein and Low Levels of Fractional Exhaled Nitric Oxide: Results From the 2007-2012 National Health and Nutrition Examination Surveys. J Clin Psychiatry. 2016 Dec;77(12):1666-71.

17. Quittner AL, Abbott J, Georgiopoulos AM, Goldbeck L, Smith B, Hempstead SE, et al. International Committee on Mental Health in Cystic Fibrosis: Cystic Fibrosis Foundation and European Cystic Fibrosis Society consensus statements for screening and treating depression and anxiety. Thorax. 2015 Oct 9; thoraxjnl-2015-207488.

18. Standards of Medical Care in Diabetes_-2013. Diabetes Care. 2013 Jan;36(Suppl 1):S11-66.

19. Lichtman JH, Bigger JT, Blumenthal JA, Frasure-Smith N, Kaufmann PG, Lespérance F, et al. Depression and coronary heart disease: recommendations for screening, referral, and treatment: a science advisory from the American Heart Association Prevention Committee of the Council on Cardiovascular Nursing, Council on Clinical Cardiology, Council on Epidemiology and Prevention, and Interdisciplinary 
Council on Quality of Care and Outcomes Research: endorsed by the American Psychiatric Association. Circulation. 2008 Oct 21;118(17):1768-75.

20. Zuckerbrot RA, Cheung A, Jensen PS, Stein REK, Laraque D, Group G-PS. Guidelines for Adolescent Depression in Primary Care (GLAD-PC): Part I. Practice Preparation, Identification, Assessment, and Initial Management. Pediatrics. 2018 Feb 26;e20174081.

21. Franx G, Kroon H, Grimshaw J, Drake R, Grol R, Wensing M. Organizational change to transfer knowledge and improve quality and outcomes of care for patients with severe mental illness: a systematic overview of reviews. Can J Psychiatry Rev Can Psychiatr. 2008 May;53(5):294-305.

22. Damschroder LJ, Aron DC, Keith RE, Kirsh SR, Alexander JA, Lowery JC. Fostering implementation of health services research findings into practice: a consolidated framework for advancing implementation science. Implement Sci. 2009;4(1):50

23. Safaeinili, N., Brown-Johnson, C., Shaw, J. G., Mahoney, M., \& Winget, M. CFIR simplified: Pragmatic application of and adaptations to the Consolidated Framework for Implementation Research (CFIR) for evaluation of a patient-centered care transformation within a learning health system. Learning Health Systems, e10201.

24.Braun V, Clarke V. Using Thematic Analysis in Psychology. Qual Res Psychol. 2006 Jan 1;3:77-101.

25. Grimshaw JM, Thomas RE, MacLennan G, Fraser C, Ramsay CR, Vale L, et al. Effectiveness and efficiency of guideline dissemination and implementation strategies. Health Technol Assess Winch Engl. 2004 Feb;8(6):iii-iv, 1-72.

26. Posner K, Brown GK, Stanley B, Brent DA, Yershova KV, Oquendo MA, et al. The ColumbiaSuicide Severity Rating Scale: Initial Validity and Internal Consistency Findings From Three Multisite Studies With Adolescents and Adults. Am J Psychiatry. 2011 Dec;168(12):1266-77.

27. Cheung AH, Zuckerbrot RA, Jensen PS, Laraque D, Stein REK, Group G-PS. Guidelines for Adolescent Depression in Primary Care (GLAD-PC): Part II. Treatment and Ongoing Management. Pediatrics. 2018 Feb 26;e20174082.

28. Saiman L, Siegel JD, LiPuma JJ, Brown RF, Bryson EA, Chambers MJ, et al. Infection Prevention and Control Guideline for Cystic Fibrosis: 2013 Update. Infect Control Hosp Epidemiol. 2014;35(S1):S167.

29. Chambers DA, Feero WG, Khoury MJ. Convergence of Implementation Science, Precision Medicine, and the Learning Health Care System: A New Model for Biomedical Research. JAMA. 2016 May 10;315(18):1941-2.

30. Boeschoten, R. E., Braamse, A. M., Beekman, A. T., Cuijpers, P., van Oppen, P., Dekker, J., \& Uitdehaag, B. M. (2017). Prevalence of depression and anxiety in multiple sclerosis: a systematic review and meta-analysis. Journal of the neurological sciences, 372, 331-341. 
31. Kobau, R., Sapkota, S., \& Zack, M. M. (2019). Serious psychological distress among adults with active epilepsy in all racial/ethnic groups and among adults with inactive epilepsy in non-Hispanic whites is significantly higher than among adults without epilepsy_US National Health Interview Survey, 2010, 2013, 2015, and 2017. Epilepsy \& Behavior, 95, 192-194.

\section{Conflict of Interest}

The authors have no conflicts of interest to declare. 
Table 1: Application of the CFIR Model to Integration of Mental Health Screening \& Treatment in CF Clinics ${ }^{1}$

\begin{tabular}{|c|c|c|}
\hline Domain Definition & Facilitators & Barriers \\
\hline $\begin{array}{l}\text { A. Intervention Characteristics } \\
\text { - Implementation of annual } \\
\text { mental health screening in CF } \\
\text { Clinics } \\
\text { - Implementation of brief, } \\
\text { evidence-based intervention in } \\
\text { Clinics } \\
\text { - Referrals within hospitals or in } \\
\text { community } \\
\text { - Follow-up of elevated scores }\end{array}$ & $\begin{array}{l}\text { Dissemination of published, } \\
\text { international guidelines and } \\
\text { algorithms } \\
\text { - Opened access to CF Foundation } \\
\text { Mental Health Listserv for } \\
\text { Psychologists/Psychiatrists to all } \\
\text { CF Providers } \\
\text { - Development and dissemination of } \\
\text { Mental Health Toolbox } \\
\text { - Screening Tools free and available } \\
\text { in all world languages }\end{array}$ & $\begin{array}{l}\text { IRB review and ethics } \\
\text { * Space and logistics in CF Clinics } \\
\text { Developing plans to develop } \\
\text { suicidal ideation } \\
\text { * Treating individuals in the } \\
\text { moderate-severe range } \\
\text { Training physicians to prescribe } \\
\text { first-line antidepressants }\end{array}$ \\
\hline
\end{tabular}

\footnotetext{
${ }^{1}$ Each domain is defined and designated alphabetically (A, B, C, etc); within each domain, the survey questions and response provide data on the variables that facilitated implementation, as well as the barriers CF Centers were working to overcome. Dark and light shading demarcate each domain.
} 


\section{B. Outer Setting}

- Resources and training for MHC's

- Evidence of elevated mental health symptoms for patients and parent caregivers

- Cosmopolitanism : CF Foundation resources \& North American CF Conference

- Peer pressure: Call to action from largest international $\mathbf{C F}$ medical conferences

- External policy and incentives: Screening guidelines adopted internationally
- TIDES study documented patient/caregiver needs

- NACFC Plenary Presentation of TIDES data

- NACFC short courses

- Funding to attend NACFC

- Regional meetings

- CFF Funding of QI Initiatives over past 12 years
* Grant funding based on center size

* Leadership involvement and support

* Hiring/Identifying appropriate personnel

* Stigma related to Mental Health screening

* Lack of mental health expertise on CF Team

* Resistance to adding new clinical practices 


\section{Inner Setting}

- Team culture
○ Multidisciplinary Team
- Type of center
○ Size of center

- Training in Quality Improvement

- Annual surveys to gather data and feedback

- CF Center Director buy-in

- CF Foundation Leadership: Executive Vice President, Chief Medical Officer

\section{Characteristics of individuals}

- Years of experience in CF care

- Professional training and background
- Multidisciplinary team meetings

- Patient/Family receptivity to screening

- Annual surveys launched via CFF and Survey Monkey

- NACFC program content on Mental Health Screening and treatment

- MHC grants funded by CFF
- Implementing parent screening of depression/anxiety

* Ethical issues related to confidentiality and storage

* Lack of knowledge and training in Mental Health issues
- Mental health toolbox

- Development and dissemination of Welcome Packet

- Dissemination of materials via Mental Health listserv

- Open discussion forum on Mental Health listserv
* Comfort with implementation of screening

* Little to no training in interventions

* Acceptability of the Screening program 


\section{E. Process of Implementation}

- MHC serves as champion of the program

- Involving and educating other stakeholders on the team

- Informing and educating patients and caregivers about the new Mental Health

Screening program

- Plan for execution of annual screening
- Training on guidelines and algorithms

- Screening Tools were free

- Development of electronic version of screening tools

- Development and dissemination of electronic scoring programs for ease of interpretation

- Storage of screening data

- Monthly mentoring program on complex cases
* Integrate new processes for screening in year 1

* New personnel integrated into the team

* Staff time

* Lack of training in counseling strategies

* Identifying appropriate therapists in the community for outside referrals

${ }^{1}$ Each domain in the CFIR Model is labeled alphabetically (A, B, etc) with alternating shading of dark and light. This column also provides definitions of the elements that make up this domain. Exemplars of both Facilitators and Barriers to implementation appear in the middle and far right columns, and were directly drawn from responses in the survey. 
Table 2. Centre Characteristics $(n=74)$

\begin{tabular}{ll}
\hline $\begin{array}{l}\text { Type of Centre } n(\%) \\
\text { Pediatric }\end{array}$ & $29(39.2 \%)$ \\
Adult & $22(29.7 \%)$ \\
$\quad$ Pediatric \& Adult & $23(31.1 \%)$ \\
Number of patients in Program (range) & $13,771(44-630)$ \\
Number 12 years + (range) & $8631(5-492)$ \\
& \\
Profession $n(\%)$ & \\
$\quad$ Social Worker & $40(54.1 \%)$ \\
$\quad$ Psychologist & $31(41.9 \%)$ \\
$\quad$ Psychiatrist & $3(4.0 \%)$ \\
Length of time within CF team $n(\%)$ & \\
Newly appointed & $13(17.6 \%)$ \\
Less than 1 year & $25(33.8 \%)$ \\
1-5 years & $20(27.0 \%)$ \\
More than 5 years & $16(21.6 \%)$ \\
\hline
\end{tabular}




\section{Table 3. Screening process}

\section{Preparedness}

Somewhat to very prepared to perform patient screening $\quad 100 \%$

Somewhat to very prepared to perform caregiver screening $\quad 79.7 \%$

Somewhat to very prepared to provide patients with the results $\quad 100 \%$

Somewhat to very prepared to provide families with the results $\quad 95.9 \%$

\section{Screening}

Initiating the screening process was somewhat easy to very easy $\quad 94.6 \%$

$\begin{array}{ll}\text { Begun screening with PHQ9 \& GAD7 100\% } & 10 \% \text {. }\end{array}$

Using recommended tools to screen caregivers* $\quad 58.6 \%$

Using the screening tools was somewhat easy to very easy $\quad 100 \%$

Scoring the screening tools was somewhat easy to very easy $100 \%$

Interpreting the screening tools was somewhat easy to very easy $\quad 98.6 \%$

Adolescents screened away from caregivers* $37.9 \%$

\section{Patient /caregiver response}

Patient response to the screening was somewhat positive to very positive $97.3 \%$ Caregiver response to the screening was somewhat positive to very $95.9 \%$ positive

\section{Training, follow-up and referrals}

Somewhat to adequately trained to counsel those scoring in mild range $\quad 98.6 \%$ Somewhat to adequately trained to provide evidence based psychological $94.6 \%$ intervention to those scoring in moderate/severe range

Providing adequate follow-up was somewhat easy to very easy $\quad 77.0 \%$

Able to make patient referrals to MH clinicians in your hospital $\quad 89.2 \%$

Started to develop a referral network of $\mathrm{MH}$ providers in the community $\quad 94.6 \%$

\section{*Answers for Pediatric Centers only}




\section{Table 4. Successes of MH screening: Themes Identified in Open-Ended Responses}

\section{Greater awareness, detection and management of MH issues for CF Providers}

- Adoption of the idea that mental health is critical to the overall health of the patient and their disease

- Improved understanding of how behavioral health impacts physical health

- Providing education about mental health to medical team (e.g., side effects of psychotropic medications)

- Development of a CF collaboration between clinical team and psychiatry and psychopharmacology

- Recognition of the value of this embedded service, both for providing higher provide quality care and improving patient outcomes and team satisfaction

- Objective data to identify and refer for treatment - people accessed services sooner

- Prevention and early identification before more serious mental health concerns arise

- Picked up on a number of patients with depression and anxiety that had been missed previously

- Identification of those at risk for suicidality and ability to provide immediate referrals

- Identification of patients with benzodiazepine dependence.

- Tracking changes in mental health symptoms over time

- Increased collaboration with community mental health providers

- Improvement in MH symptoms in those who are screened and treated

- CF team serving as model for mental health screening in other subspecialty clinics (e.g., asthma) 


\section{Patients more able to discuss $\mathrm{MH}$ issues - destigmatizing MH concerns}

- Patients have been receptive to screening and view their physical and mental health as linked

- Screening has helped normalize and destigmatize mental health issues even within CF team!

- Created a more open dialogue about mental health issues

- Patients who were resistant to mental health treatment in the past are now seeking care

- Identification of depression and anxiety in patients who would not vocalize these symptoms

- More patients have opened-up about and expressed interest in mental health services

- Opportunity for patients, parents and providers to discuss the importance of behavioral health issues in CF care

- Increased patient awareness of support available to them in the CF center and community

\section{Gratitude and support from families / patients for addressing $\mathrm{MH}$}

- Patients and families have embraced the screening process and often express "relief" that emotional health and well-being can be addressed

- Families expressed gratitude that CF teams are acknowledging the challenges and stressors for caregivers

- Offering patients and families options for treating anxiety and depression rather than learning to "live with it".

- Interest and acceptance of screening for caregivers; Parents feeling "heard"

- Very appreciative that we are listening to them differently; integrating mental health into physical health visits 


\section{Table 5. Barriers/Ethical issues of MH screening: Themes Identified in Content Analyses of Open-Ended Responses}

\section{'Legal' aspects of screening adult caregivers in a pediatric department and storage of} caregiver data

- Dilemma that parent is not the "identified patient;"

- Need to consider the ethical and legal/risk management implications of screening adult caregivers in a pediatric setting

- Confusion about where to record parent scores; inclusion in child's EMR?

- Sharing information with team; how much to share?

- CF parent community expressed concerns about storage of their MH data and privacy

\section{Difficulty with Logistics, Follow-up and Referrals}

- Patient perceptions indicated that clinics very busy and this might add time

- Wanting some advanced notice about the onset of screening

- Lack of psychiatry support

- Challenges with logistics when someone is potentially suicidal; having adequate training and preparation for this

- Problems with referrals to mental health practitioners because of insurance barriers

- Lack of clinical time to follow up for those with elevations in depression and anxiety

- Difficulty identifying providers in the community

- Space to screen adolescent separately from parent

- Challenge of keeping adolescent screening data confidential and not sharing with parents

- Patient's not following through with interventions and/or referrals made based on their scores 


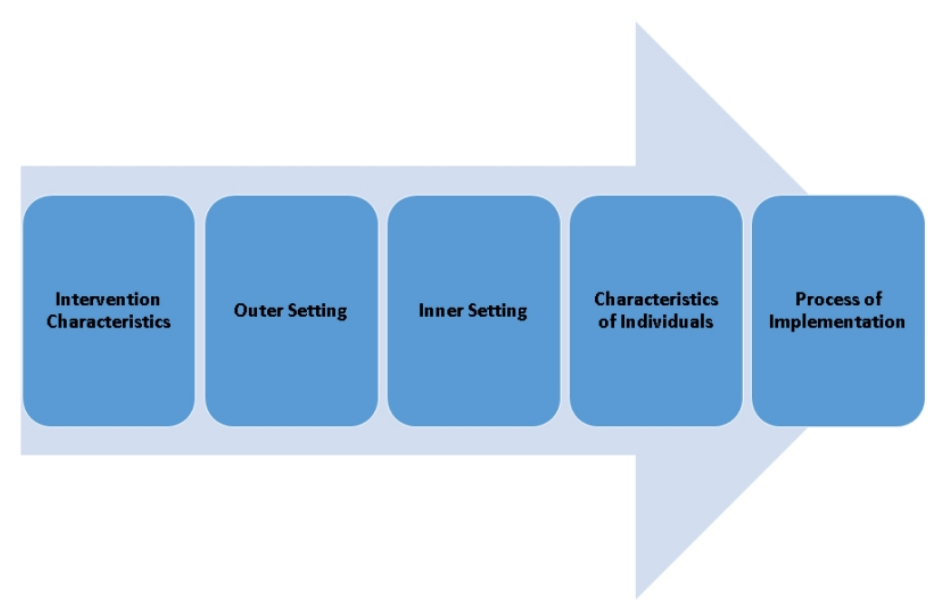

The CFIR Model: Major Domains and Exemplar Items $338 \times 190 \mathrm{~mm}(96 \times 96 \mathrm{DPI})$ 


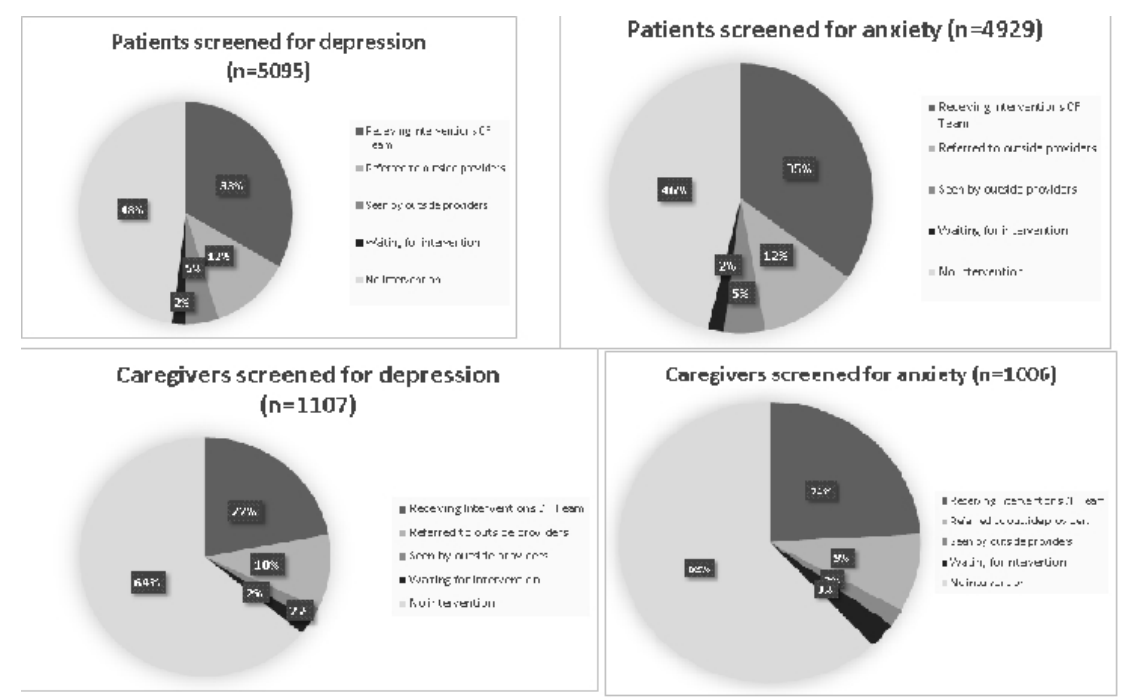

Interventions \& Referrals Following Elevated Screening Scores $228 \times 128 \mathrm{~mm}(96 \times 96 \mathrm{DPI})$ 


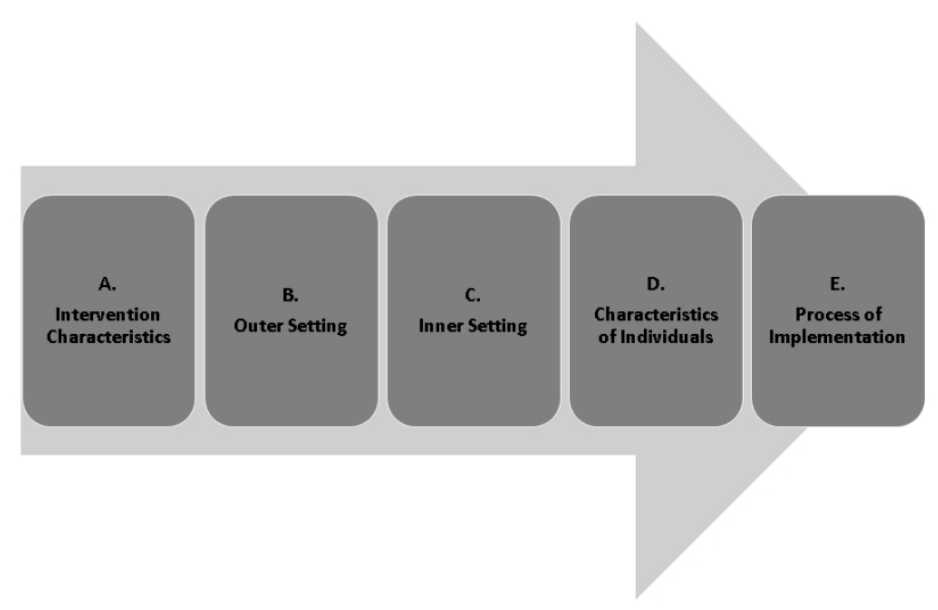

Consolidated Framework for Research Implementation (CFIR): Domain definitions linked to Facilitators and Barriers to Implementation

$338 \times 190 \mathrm{~mm}(96 \times 96 \mathrm{DPI})$ 